\title{
- 保护论坛・
}

\section{保护地旅游公路的野生动物通道 设计原则与技术参数}

\author{
余小林 ${ }^{1,2}$ 周友兵 ${ }^{*}$ 徐文婷 ${ }^{1}$ 谢宗强 ${ }^{1}$ \\ 1 (中国科学院植物研究所植被与环境变化国家重点实验室, 北京 100093) \\ 2 (中国科学院大学, 北京 100049)
}

\begin{abstract}
摘要: 保护地以其丰富的生物多样性和优美的自然环境为生态旅游的开展提供了基础条件。近年来, 保护地的生 态旅游与旅游道路建设得到了飞速发展。旅游公路的修建，在促进经济发展的同时，也带来了野生动物致死、基 因隔离、栖息地丧失、生境破碎化等一系列生态问题。因此设立合适的野生动物通道作为一种有效方式, 成为缓解 公路对野生动物负面影响的主要途径。本文基于动物通道相关研究, 提出通道设计应遵从针对性、科学性、持续有 效性、可行性四条原则, 道路生态学与保护生物学相关理论、保护地管理法规与管理规划、关键物种或类群生态学 特性与栖息地现状以及沿线地形地貌特征都应作为通道设置的参考依据; 并从通道建设的数量、位置、类型、尺寸、 表面设计、配套设施以及后期监测等方面提出了通道建设的技术参数。为长期有效地发挥野生动物通道的生态功能, 建议制定通道建设技术规范，细化通道技术参数，积极开展科研监测，以缓解道路对野生动物的影响。
\end{abstract}

关键词: 道路生态学, 旅游公路, 持续有效性, 野生动物保护, 技术参数, 生态旅游意识

\section{Principle and technical parameters for designing wildlife crossings on tourism roads in protected areas}

\author{
Xiaolin $\mathrm{Yu}^{1,2}$, Youbing $\mathrm{Zhou}^{1 *}$, Wenting $\mathrm{Xu}^{1}$, Zongqiang Xie ${ }^{1}$ \\ 1 State Key Laboratory of Vegetation and Environmental Change, Institute of Botany, Chinese Academy of Sciences, \\ Beijing 100093 \\ 2 University of Chinese Academy of Sciences, Beijing 100049
}

\begin{abstract}
Protected areas are crucially important for safeguarding biodiversity, and can provide a sustainable means for generating local community income through nature-based ecotourism. Although tourism roads are thus prerequisites for the development of protected areas, they have a negative impact on wildlife, through direct traffic casualties (mortality), and habitat loss and fragmentation, which can lead to genetic isolation. Wildlife crossings have been proven to be one of the most important ways to alleviate these negative effects. Here, we collected data by completing a literature review, and proposed that pertinence, science, sustainability and validity, and feasibility should be regarded as the four main principles for designing wildlife crossing. A better understanding of the theory regarding road ecology and conservation biology, protection regulations and management plans of protected areas, bio-ecological characteristics of key species, and wildlife habitat and topography along tourism roads may contribute enormously to determine the number, type, location, size and monitoring of wildlife crossings. Furthermore, we suggest that wildlife crossings will work more effectively when they are well designed and constructed, under continuous monitoring and regulation by the administration, and in concert with awareness of both eco-tourists and tour operators driving within the reserve. The use of these crossings may contribute significantly to improving the balance between ecotourism and the conservation of wildlife in protected areas.
\end{abstract}

Key words: road ecology, tourism road, sustainability and validity, wildlife conservation, technical parameters, eco-tourism consciousness 
随着社会经济的飞速发展和人民生活水平的 提高, 大众旅游得到迅猛发展(章小平和朱忠福, 2007)。保护地由于有独特的生态服务功能, 常成为 公众休闲的目的地(Liu et al., 2001; He et al., 2008)。 为更好地保护和维护生物多样性、自然及文化资源, 世界自然保护联盟(IUCN)基于不同的管理目标将 保护地划分为6类, 并划分出核心区、缓冲区和实验 区进行分区管理。地方政府和保护区管理部门则希 望在不影响保护的前提之下, 在保护地(除严格的 自然保护区(strict nature reserves))的缓冲区和实验 区将科学研究、教育、生产和生态旅游活动进行有 机结合, 使其生态、社会和经济效益得到充分发挥 (彭晓东, $2007^{(1)}$; 郭进辉, 2008)。

对于地处偏远的保护地而言, 开展生态旅游的 第一限制因素往往是公路建设。旅游公路作为保护 地最重要的道路类型之一, 是将保护地旅游景点进 行有效连接, 以方便开展生态旅游活动而修建的道 路。由于旅游公路往往修建于生物多样性丰富、生 态环境脆弱敏感的区域, 因此在勘测、设计、施工 中对环境保护的要求远远高于其他公路。已有研究 表明, 公路修建对野生动物造成的影响包括: 道路 致死(Forman \& Alexander, 1998; Kong et al., 2013)、 种群移动扩散受限 (Alexander \& Waters, 2000; McDonald \& Clair, 2004)、种群定居率降低、灭绝风 险增加(Mader, 1984)、基因交流受阻(Corlatti et al., 2009)、生境破碎化(Mader, 1984; Zhou et al., 2013) 等一系列生态问题。当公路穿越自然保护区等生物 多样性丰富、生态敏感脆弱区时, 这种影响尤为严 重。为缓解公路建设带来的不利影响, 野生动物通 道 (即为帮助动物安全通过公路或铁路而特别修建 的工程设施, 如天桥、高架桥、涵洞等)作为缓解道 路负面生态效应的一项有效措施开始得到应用与 发展 (Forman \& Alexander, 1998; Alexander \& Waters, 2000; Corlatti et al., 2009)。

早在 20 世纪 $50 、 60$ 年代, 欧洲就开始关注铁路 和公路对野生动物的影响及其缓解措施(Forman \& Alexander, 1998)。随后, 法国、德国、瑞士、瑞典、 西班牙、加拿大、澳大利亚等发达国家开始将动物 通道广泛应用于公路建设中(Clevenger \& Waltho, 2000; Bank et al., 2002; Mata et al., 2008), 并对通道

(1) 彭晓东 (2007) 长白山生态旅游发展问题研究. 吉林大学硕士学位 论文, 长春.
的功能(如公路致死效应的缓解、生境破碎化的改善 等)开展了广泛研究(Clevenger \& Waltho, 2005; Mata et al., 2008)。我国则起步较晚(铁道部野生动 物保护考察团, 2003; 国家林业局, 2012), 自2003年 青藏铁路建设引入野生动物通道这一概念以来, 仅 在青藏铁路、云南思茅-小预养高速公路以及吉林 长白山设立了野生动物通道(Pan et al., 2009; Kong et al., 2013), 并对其进行了长期监测(Yang \& Xia, 2008)。目前, 已监测到藏羚羊(Pantholops hodgsoni) 通过通道次数高达2,952次(Yang \& Xia，2008), 亚 洲象(Elephas maximus)对下跨式通道的利用率达到 44\% (Pan et al., 2009), 狍(Capreolus pygargus)、野 猪(Sus scrofa) 等野生动物也已逐步开始利用通道 (Kong et al., 2013), 表明这些通道的建设已初步达 到预设目的。然而, 对生物多样性丰富的保护地内 的旅游公路却鲜有报道。

本文对公路野生动物通道的研究和实践现状 进行了分析, 进而总结了保护地旅游公路上野生动 物通道设置的原则、依据与技术参数, 为在保护地 旅游公路上构建高效的野生动物“生命通道”, 同时 为缓解由旅游公路修建和运营导致的动物死亡、栖 息地破碎化等生态问题提供科学依据。

\section{1 野生动物通道建设的原则与依据}

\section{1 设计原则}

(1)针对性原则。针对目标物种或类群设计专门 的野生动物通道(国家林业局, 2012; 李玉强等, 2013), 可以更好地保证其利用率和有效性。针对的 目标物种可以是保护地内被列入IUCN红色名录极 危 $(\mathrm{CR}) 、$ 濒危 $(\mathrm{EN})$ 、易危 $(\mathrm{VU})$ 的野生动物, 《濒危 野生动植物种国际贸易公约》(CITES) (http://www. cites.org/) 附录I和附录II中的野生动物, 国家和地方 重点保护的野生动物, 也可以是保护地的关键物种 和类群(Beckmann et al., 2010)。还应针对目标物种 或类群的生物生态学特性, 对不同类型动物的通道 进行针对性设计。如大型有蹄类、树栖类动物更适 合上跨式通道, 而两栖爬行类则倾向下钻式通道 (Clevenger \& Huijser, 2011)。

(2)科学性原则。设计通道前应了解道路阻隔效 应及其缓解措施等道路生态学理论以及威胁生物 多样性的因素、保护地的分区管理、种群灭绝机制、 种群生存力分析等保护生物学理论(Primack和马克 
平, 2009; Beckmann et al., 2010), 并熟悉相关法律 法规(如环境保护法、野生动物保护法、公路法等) 的要求。还应结合目标物种的分布、栖息地特征、 运动与迁徙路线等生态学信息, 辅以野外调查数 据, 科学确定通道设计的主要技术参数(Beckmann et al., 2010; 李玉强等, 2013)。

(3)持续有效性原则。在通道建成后, 应加强对 通道的维护管理, 保证通道功能的持续发挥。同时, 加强监控影响通道持续利用的因素, 如目标物种的 数量特征、习性、栖息地条件以及附近人类活动(王 云等, 2007)。还应设计科学系统的实验对通道的有 效性进行评估(Yang \& Xia, 2008; Mata et al., 2008)。

(4)可行性原则。应在确保通道的长期安全性和 持久稳定性的前提下, 充分考虑经济和技术上的可 行性, 在满足保护目标的基础上尽可能降低建设成 本(王云等, 2007)。

\section{2 设计依据}

(1)道路生态学和保护生物学的基础理论。道路 生态学研究显示, 目前道路的修建已引起野生动物 死亡、种群移动与扩散受限、动物基因交流受阻等 一系列生态问题 (Forman \& Alexander, 1998; Spellerberg, 1998; Corlatti et al., 2009)。保护生物学 理论认为在孤立的栖息地斑块间建立野生动物通 道可以维持或提高动物在斑块间的扩散水平, 从而 维持目标物种的基因流和种群生存力(Forman \& Alexander, 1998)。即使野生动物对通道的利用率相 对较低, 也能降低小种群的灭绝风险(Taylor \& Goldingay, 2009)。了解道路生态学、保护生物学相 关理论, 可为确定通道建设与否、通道建设目的与 意义提供依据。

(2)保护地管理法规与管理规划。了解保护地管 理法规中与野生动物保护措施相关的条款, 便于充 分、细致地考虑项目建设对生态环境和生物多样性 的影响(铁道部野生动物保护考察团, 2003); 了解 保护地管理规划中关于旅游容量、道路等基础设施 建设、分区管护、监测与科研、投资计划等相关内 容, 有利于掌握保护地本底情况, 为通道类型、位 置选择及后期管理提供依据。

(3)关键类群的生态学特性及其栖息地现状。旅 游公路沿线关键物种或类群的生态学特性及栖息 地现状是有效设计通道的依据。主要包括关键物种 的种群分布、大小、移动与迁移路线、食性等生态
学特征及其栖息地的保护现状; 同时，还应兼顾考 虑与关键物种同域动物的生态学特征及栖息地特 征，为确定通道的数量、位置、类型、尺寸等技术 参数提供参考依据。

(4)沿线的地形地貌特征。地形地貌特征是影响 通道位置、类型选择的重要因素(Clevenger \& Huijser，2011)。通过收集自然保护区地形图、野生 动物分布图、迁移扩散图, 并结合野外考察的方式, 了解保护区旅游公路沿线的地形地貌特征, 为通道 位置、类型选择提供依据。

\section{2 野生动物通道设计技术参数}

在野生动物通道设计时，应尽可能科学地考虑 通道建设的数量、位置、类型、尺寸、表面设计、 配套设施以及后期监测等相关方面的内容。

(1)通道数量。可综合考虑保护动物的类型、数 量与迁移能力，以及保护地的经济状况、人类活动、 旅游公路沿线地形特征等因素，确定不同旅游公路 段的通道设置间距。目前关于动物通道设置的间距 并没有明确的规定，也没有具体计算公式。根据现 有资料(Clevenger \& Waltho, 2005; Dodd et al., 2007; Clevenger \& Huijser, 2011), 高速公路上动物通道设 置的间距从 $1.5 \mathrm{~km}$ 到 $6.0 \mathrm{~km}$ 不等。但具体设计时, 要 考虑到目标物种或类群的分布与行为特性。

(2)通道位置。通道的位置关系着其能否被动物 有效利用(Clevenger \& Waltho, 2000)。应充分结合保 护地地形、野生动物的分布、迁移扩散路线、公路 致死数据等相关资料, 选择合理的通道位置(李玉 强等, 2013)。然而有研究显示, 基于道路致死数据 选择的通道位置并不是最佳选择(Clevenger，2012)， 这是由于保护地可能会存在数据缺乏的情况，可以 通过基于专家经验的栖息地模型、快速评估、走访 调查等方法, 综合分析确定可能的合适位置 (Clevenger \& Huijser, 2011)。

(3)通道类型。通道类型的选择主要受到野生动 物栖息地质量、地形因素及建设成本的限制 (Clevenger \& Huijser, 2011; Ruediger \& Basting, 2012), 包括上跨式和下钻式两种通道类型。上跨式 通道(附录1)多设于地形较为平缓的或“U”形地段, 主要适合喜开阔环境的大型有蹄类动物、食肉动 物、树栖或半树栖动物通过(Glista et al., 2009; Clevenger \& Huijser, 2011; Kong et al., 2013)。下钻 
式通道(附录2)多依地形而设, 多建于平缓、隆起、 湿地、水域等区域, 常以涵洞形式存在, 主要适合 小型哺乳动物、两栖类、爬行类动物通过(Clevenger \& Huijser, 2011; Cramer et al., 2012)。研究显示, 棕 熊(Ursus arctos)、欧洲马鹿(Cervus elaphus)、狼 (Canis lupus)更喜欢利用上跨式通道, 而美洲黑熊 (Ursus americanus)、美洲狮(Puma concolor)则没有 表现出明显的偏好 (Clevenger \& Waltho，2000; Clevenger \& Waltho, 2005; Clevenger, 2012)。对于水 渠旁高速公路上的下钻式通道一一涵洞, 小型哺乳 动物对其的利用率高达 $100 \%$, 两栖动物利用率达 75\%(Veenbaas \& Brandjes, 1999)。同时, 由于保护地 生物多样性丰富, 可以建立多种类型的通道, 以降 低公路的阻隔效应(Mata et al., 2008)。

(4)通道尺寸。已有研究表明通道尺寸影响通道 的利用率及有效性(Clevenger \& Waltho, 2000; Ng et $a l .$, 2004), 但通道尺寸没有固定标准, 应结合考虑通 道用途、目标物种或类群的个体大小、行为特性等 因素。上跨式通道宽度应不小于 $10 \mathrm{~m}$, 而宽度大于 50 $\mathrm{m}$ 时可满足绝大部分动物通过(Bank et al., 2002)。在 地形和经济允许的条件下, 越宽越好。在德国、法国 建成的上跨式通道最宽达到了 $870 \mathrm{~m}$ (Bank et al., 2002)。下钻式通道尺寸相对上跨式通道偏小，宽 10 $\mathrm{m}$ 、高 $4 \mathrm{~m}$ 的通道基本上能满足所有利用该类型通道 的动物通过(Clevenger \& Huijser, 2011)。已有资料显 示(Clevenger \& Waltho, 2005), 在建设通道时应综合 考虑其尺寸，把开度(即宽 $\times$ 高 $/$ 长) (Reed \& Ward, 1987)这一概念运用到实践中, 开度可能比通道整体 大小更为重要(Clevenger \& Waltho, 2005)。

(5)通道表面设计。上跨式通道表面的基质和土 壤应该就地取材, 使其尽可能与周围环境一致, 铺 设厚度应该考虑能给植物提供足够的水分和支撑 (Clevenger \& Huijser, 2011)。同时, 应在通道上种植 目标保护动物所偏好的植物, 以降低其心理恐惧, 顺利通过通道。下钻式通道则应考虑到目标物种的 种类。如果是两栖类, 则应修建小沟渠引导流水流 入通道, 保持通道的潮湿(Jackson, 1996); 若是小型 有蹄类动物, 则应考虑通道的排水性, 以免受到暴 雨影响(Clevenger \& Huijser, 2011)。

(6)监测系统。为了配合保护区的科研工作, 可 在通道上安装红外监测系统, 辅以足迹追踪(如sand beds、snow tracking)等方法(Bank et al., 2002), 对通
道的合理性、使用效率进行检验，并对不合理之处 及时调整。更为重要的是可以监测动物通过通道的 行为特性, 这对于研究动物对通道的适应时间、适 应机制以及动物穿越通道的决定因素等科学问题 具有重要意义(Clevenger, 2012; Wang, 2014)。

(7)配套设施。应在通道两侧设置栅栏以引导动 物进入通道(Leblond et al., 2007), 阻止其进入旅游 公路而发生车祸。在通道位置前后设计交通标志牌 或季节性警示牌(王云等, 2007; Beckmann et al., 2010), 提醒司机减速慢行。虽然目前缺乏对树立警 示牌的有效性研究, 但其有效性评估已引起广泛关 注(Kong et al., 2013)。此外, 如果通道远离水源, 还 应修建简易蓄水池用于植物的浇灌(Clevenger \& Huijser, 2011)。

\section{3 对策与建议}

\section{1 制定技术规范, 完善法律体系}

在设计旅游公路时, 应加强跨学科领域的合 作, 工程和生态学领域的专家应合作设计有效的公 路建设方案(Kong et al., 2013), 并向环保部门提交 环境影响评价报告(铁道部野生动物保护考察团, 2003)。线路选择上尽量避免穿越珍濒野生动物分布 区, 实在无法避免时, 应将公路阻隔效应的缓解措 施纳入旅游公路设计方案。如设立野生动物通道、 安装动物监测系统(animal detection systems)和地音 探测器(geophones)、建设电子垫与栅栏(electric mats and fencing)等都是比较有效的措施(Gray，2009; Beckmann et al., 2010)。同时，应完善相关的法律法 规, 成立监督委员会, 为旅游公路设计和建设过程 中将建设野生动物通道纳入规范提供法律保障。

我国2015年1月1日起实施的《中华人民共和国 环境保护法》(2014年修订版) (http://www.gov.cn/ xinwen/2014-04/25/content_2666328.htm) 明确规定 应对重点生态功能区、生态环境敏感脆弱区进行严 格保护，合理开发利用自然资源，保护生物多样性， 建设对环境有影响的项目时应该依法进行环境影 响评价。《公路法 (第二次修正)》(http://www.gov.cn/ banshi/2005-08/23/content_25592.htm)提出在公路建 设时应特别注意环境保护。《野生动物保护法》 (http://news.xinhuanet.com/zhengfu/2004-08/30/conte nt_1925402.htm) 则对珍贵、濒危野生动物保护提出 了更为严格的要求。2012年7月1日，我国颁布了首 
部针对野生动物通道设计的法规《陆生野生动物廊 道设计技术规程》(国家林业局, 2012)。但这项技术 规程对于保护地而言相对宽泛, 缺乏针对性。因此, 应根据现有法律法规, 结合保护地具体情况, 制定 针对性强、可操作性强的《旅游公路野生动物通道 设置技术规范》。

\section{2 加强基础调研, 细化技术参数}

应加大科研力度, 加强对旅游公路建设前的科 学调查和研究, 避免通道建设的技术性错误。一方 面, 对保护地的动植物分布规律、动物活动迁徙路 线、地形地貌等基础信息作全面细致的调查(李玉强 等, 2013), 设计科学合理的技术路线, 选择合适的 通道位置; 另一方面, 由于通道的类型、尺寸、体 量、表面设计等是其利用率的重要影响因素(Wang, 2014), 因此加强通道建设的技术研究尤为重要。 Cramer等(2012)根据结构和功能将通道分为7类, 并 对其进行尺寸、适合通过的动物进行了简单阐述。 对班夫国家公园通道的监测研究表明, 鹿(Odocoileus sp.)最喜欢利用陆桥, 其次是金属涵洞、箱形 涵洞; 欧洲马鹿最喜欢用金属涵洞，同时也使用陆 桥(Clevenger et al., 2009)。然而, 目前对于通道的光 线、温度、湿度等的研究则是屈指可数, 相关研究 仍亟待加强。

\section{3 开展科研监测，搭建动物信息化平台}

持续的后期监测可以对动物通道有效性进行 实时评价(Mata et al., 2008; Yang \& Xia, 2008), 并 不断改进(Dussault et al., 2007; 李斌等, 2012 ${ }^{\circledR}$ ), 使 其更好地满足动物的通行。如科学家对加拿大横贯 公路(Trans-Canada Highway)穿越班夫国家公园段 修建的38座下跨式通道和6座上跨式通道进行长达 15 年的监测结果显示, 动物通过通道次数高达 198,811 次, 其中鹿和欧洲马鹿分别占 $62 \%$ 和 $19 \%$ (Clevenger, 2012)。同时, 还可以通过监测数据 获得通过通道的动物的种类、穿越通道时的行为等 资料(Bank et al., 2002)。持续的监测辅以后期科学 的实验设计, 也为研究影响动物对旅游公路上通道 有效利用的决定因素、动物对通道的适应机制等科 学问题提供了资料(Wang, 2014)。

\section{4 提高生态旅游意识, 实现人一路-自然和谐共存} 生态旅游被誉为是“回归大自然之旅”、“健康之

(1) 李斌 (2012) 韶赣高速公路野生动物通道两栖动物保护效果及改进 措施研究. 北京林业大学硕士学位论文, 北京.
旅”，旅游公路的修建为更多人到保护地旅游、欣赏 自然美景、接触珍稀濒危动植物提供了可能。然而, 生态旅游的发展也带来了噪音污染、大气污染、生 活垃圾等一系列问题, 这都是威胁野生动物生存和 繁衍的重要因素(Wang, 2014)。因此, 应加强生态保 护宣传教育，提高全民环境意识和生态旅游意识， 尤其是在旅游公路沿线生态环境脆弱区、珍稀濒危 野生动物的分布区以及野生动物通道处应完善警 示标志, 提醒司机和游客谨慎慢行, 减少鸣笛, 避 免大声喧哗, 以减小对野生动物的干扰和动物交通 事故发生的可能(Kong et al., 2013)。

\section{参考文献}

Alexander SM, Waters NM (2000) The effects of highway transportation corridors on wildlife: a case study of Banff National Park. Transportation Research Part C: Emerging Technologies, 8, 307-320.

Bank FG, Irwin CL, Evink GL, Gray ME, Hagood S, Kinar JR, Levy A, Paulson D, Ruediger B, Sauvajot RM, Scott DJ, White P (2002) Wildlife habitat connectivity across European highways. Technical Report FWHA-PL-02-011. US Department of Transportation, Washington, DC.

Beckmann JP, Clevenger AP, Huijser MP, Hilty JA (2010) Safe Passages: Highways, Wildlife, and Habitat Connectivity. Island Press, Washington, DC.

Clevenger AP, Waltho NG (2000) Factors influencing the effectiveness of wildlife underpasses in Banff National Park, Alberta, Canada. Conservation Biology, 14, 47-56.

Clevenger AP, Waltho NG (2005) Performance indices to identify attributes of highway crossing structures facilitating movement of large mammals. Biological Conservation, 121, 453-464.

Clevenger AP, Huijser MP (2011) Wildlife Crossing Structure Handbook: Design and Evaluation in North America. Technical Report FHWA-CFL/TD-11-003. US Department of Transportation, Washington, DC.

Clevenger AP (2012) 15 years of Banff research: what we've learned and why it's important to transportation managers beyond the park boundary. In: Proceedings of the 2011 International Conference on Ecology and Transportation (ICOET 2011), pp. 433-447. Seattle, Washington, United States, August 21-25, 2011.

Corlatti L, Hacklaender K, Fredy-Roos F (2009) Ability of wildlife overpasses to provide connectivity and prevent genetic isolation. Conservation Biology, 23, 548-556.

Cramer P, Kintsch J, Jacobson S (2012) Maintaining wildlife connectivity across roads through tested wildlife crossing designs. In: Proceedings of the 2011 International Conference on Ecology and Transportation (ICOET 2011), pp. 448-460. Seattle Washington, United States, August 21-25, 2011.

Dodd NL, Gagnon JW, Boe S, Manzo A, Schweinsburg RE (2007) Evaluation of measures to minimize wildlife-vehicle collisions and maintain permeability across highways: Arizona 
Route 260. Final Report 540. FHWA-AZ-07-540. Arizona Department of Transportation, Phoenix, Arizona, USA.

Dussault C, Ouellet JP, Laurian C, Courtois R, Poulin M, Breton L (2007) Moose movement rates along highways and crossing probability models. Journal of Wildlife Management, 71, 2338-2345.

Forman RTT, Alexander LE (1998) Roads and their major ecological effects. Annual Review of Ecology and Systematics, 29, 207-231.

Glista DJ, DeVault TL, DeWoody JA (2009) A review of mitigation measures for reducing wildlife mortality on roadways. Landscape and Urban Planning, 91, 1-7.

Gray M (2009) Advances in wildlife crossing technologies. Public Roads, 73(2), 1-14.

Guo JH (郭进辉) (2008) Study on Community-Based Forest Eco-Tourism in Wuyishan Nature Reserve (基于社区的武 夷山自然保护区森林生态旅游研究). $\mathrm{PhD}$ dissertation, Beijing Forestry University, Beijing. (in Chinese with English abstract)

He GM, Chen XD, Liu W, Bearer S, Zhou SQ, Cheng LY, Zhang HM, Ouyang ZY, Liu JG (2008) Distribution of economic benefits from ecotourism: a case study of Wolong Nature Reserve for giant pandas in China. Environmental Management, 42, 1017-1025.

Kong Y, Wang Y, Guan L (2013) Road wildlife ecology research in China. Procedia-Social and Behavioral Sciences, 96, 1191-1197.

Liu JG, Linderman M, Ouyang ZY, An L, Yang J, Zhang HM (2001) Ecological degradation in protected areas: the case of Wolong Nature Reserve for giant pandas. Science, 292, 98-101.

Li YQ (李玉强), Xing SH (邢韶华), Liu SQ (刘生强), Luo AD (罗爱东), Cui GF (崔国发) (2013) Designing method of terrestrial wild life path. Journal of Beijing Forestry University (北京林业大学学报), 35(6), 137-143. (in Chinese with English abstract)

Mader HJ (1984) Animal habitat isolation by roads and agricultural field. Biological Conservation, 29, 81-96.

Mata C, Hervás I, Herranz J, Suárez F, Malo JE (2008) Are motorway wildlife passages worth building? Vertebrate use of road-crossing structures on a Spanish motorway. Journal of Environmental Management, 88, 407-415.

McDonald WR, Clair CCS (2004) The effects of artificial and natural barriers on the movement of small mammals in Banff National Park, Canada. Oikos, 105, 397-407.

Ng SJ, Dole JW, Sauvajot RM, Riley SP, Valone TJ (2004) Use of highway undercrossings by wildlife in southern California. Biological Conservation, 115, 499-507.

Pan W, Lin L, Luo A, Zhang L (2009) Corridor use by Asian elephants. Integrative Zoology, 4, 220-231.

Primack RB, Ma KP (马克平) (2009) A Primer of Conservation Biology (保护生物学简明教程). Higher Education
Press, Beijing. (in Chinese)

Reed DF, Ward AL (1987) Efficacy of methods advocated to reduce deer-vehicle accidents: research and rationale in the USA. In: Proceedings of the Highway and Wildlife Relationships, pp. 285-293. Strasbourg, France, June 5-7, 1985.

Ruediger WBC, Basting PB (2012) High, wide and handsome: a review of wildlife and aquatic crossing technology (2001-2011). In: Proceedings of the 2011 International Conference on Ecology and Transportation (ICOET 2011), pp. 461-474. Seattle, Washington, United States, August 21-25, 2011.

Spellerberg IAN (1998) Ecological effects of roads and traffic: a literature review. Global Ecology and Biogeography, 7, 317-333.

State Forestry Administration of the People's Republic of China (国家林业局) (2012) Technical Regulation for Terrestrial Wildlife Corridor Design (陆生野生动物廊道设 计技术规程). China Standards Press, Beijing. (in Chinese)

Taylor BD, Goldingay RL (2009) Can road-crossing structures improve population viability of an urban gliding mammal? Ecology and Society, 14, 13-32.

Veenbaas G, Brandjes J (1999) Use of fauna passages along waterways under highway. In: Proceeding of the International Conference on Wildlife Ecology and Transportation. Florida Department of Transportation, Tallahassee.

Wang J (2014) Effectiveness of Wildlife Crossing Structures on Providing Habitat Connectivity for Wild Animals. PhD dissertation, the University of British Columbia, New York.

Wang Y (王云), Li HF (李海峰), Cui P (崔鹏), Wu H (吴浩) (2007) A study on wildlife passage along highway in Wolong National Nature Reserve. Highway (公路), (1), 99-104. (in Chinese with English abstract)

Wildlife Conservation Delegation of the Ministry of Railways (铁道部野生动物保护考察团) (2003) Railway wildlife crossing and ecological protection. Railway Labor Safety and Health and Environment Protection (铁道劳动安全卫 生与环保), 30(1), 8-11. (in Chinese)

Yang Q, Xia L (2008) Tibetan wildlife is getting used to the railway. Nature, 452, 810-811.

Zhang XP (章小平), Zhu ZF (朱忠福) (2007) An analysis of tourism environment capacity in Jiuzhaigou Scenic Area. Tourism Tribune (旅游学刊), 22(9), 50-57. (in Chinese with English abstract)

Zhou YB, Buesching CD, Newman C, Kaneko Y, Xie ZQ, Macdonald DW (2013) Balancing the benefits of ecotourism and development: the effects of visitor trail-use on mammals in a protected area in rapidly developing China. Biological Conservation, 165, 18-24.

(责任编委: 蒋志刚 责任编辑: 时意专)

\section{附录 Supplementary Material}

附录1 上跨式野生动物通道效果示意图

Appendix 1 Schematic diagram of wildlife overpass http://www.biodiversity-science.net/fileup/PDF/w2015-022-1.pdf
附录2 下钻式野生动物通道效果示意图

Appendix 2 Schematic diagram of wildlife underpass http://www.biodiversity-science.net/fileup/PDF/w2015-022-2.pdf 
余小林, 周友兵, 徐文婷, 谢宗强. 保护地旅游公路的野生动物通道设计原则与技术参数. 生物多样性, 2015, 23 (6)：824-829. http://www. biodiversity-science. net/CN/10. 17520/biods. 2015022

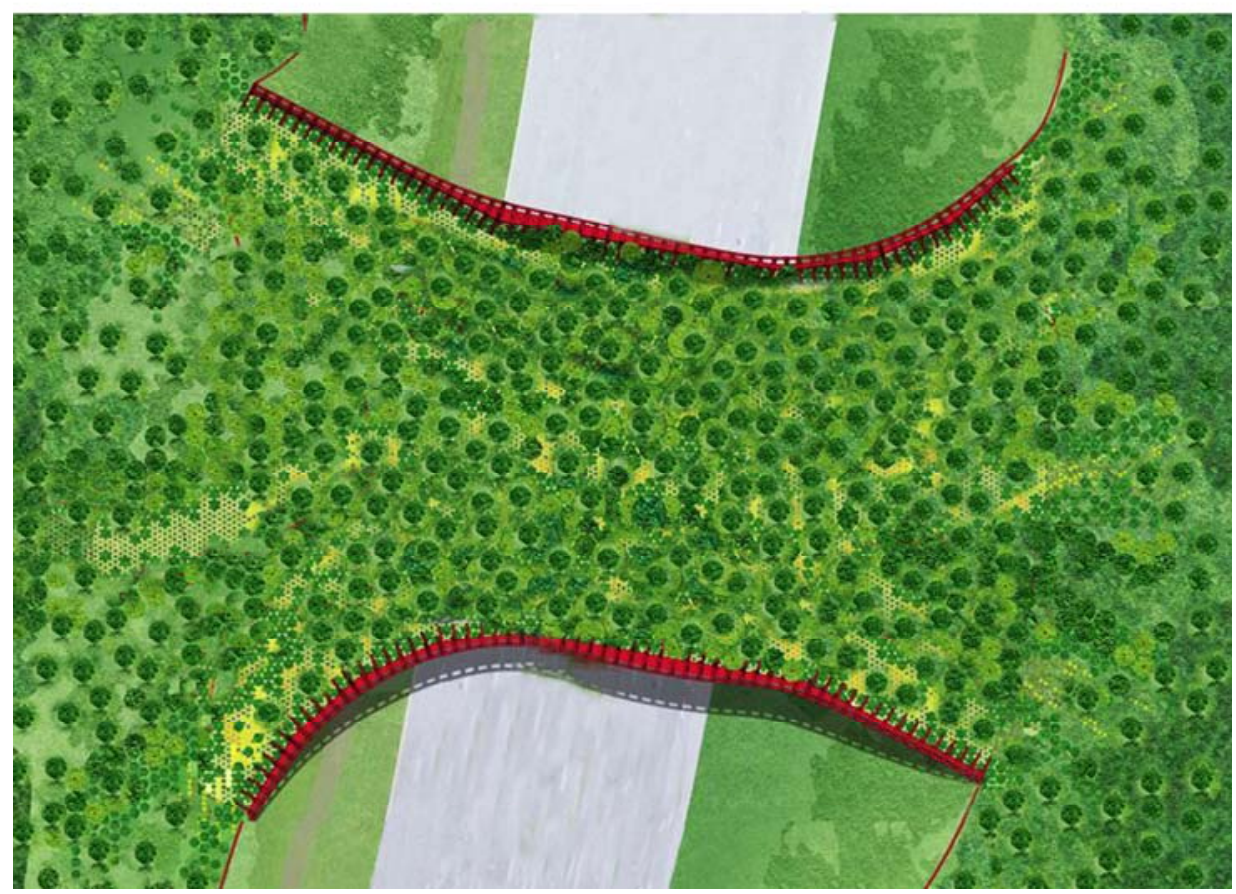

附录1 上跨式野生动物通道效果示意图

Appendix 1 Schematic diagram of wildlife overpass 
余小林, 周友兵, 徐文婷, 谢宗强. 保护地旅游公路的野生动物通道设计原则与技术参数. 生物多样性, 2015, 23 (6)：824-829. http://www. biodiversity-science. net/CN/10. 17520/biods. 2015022

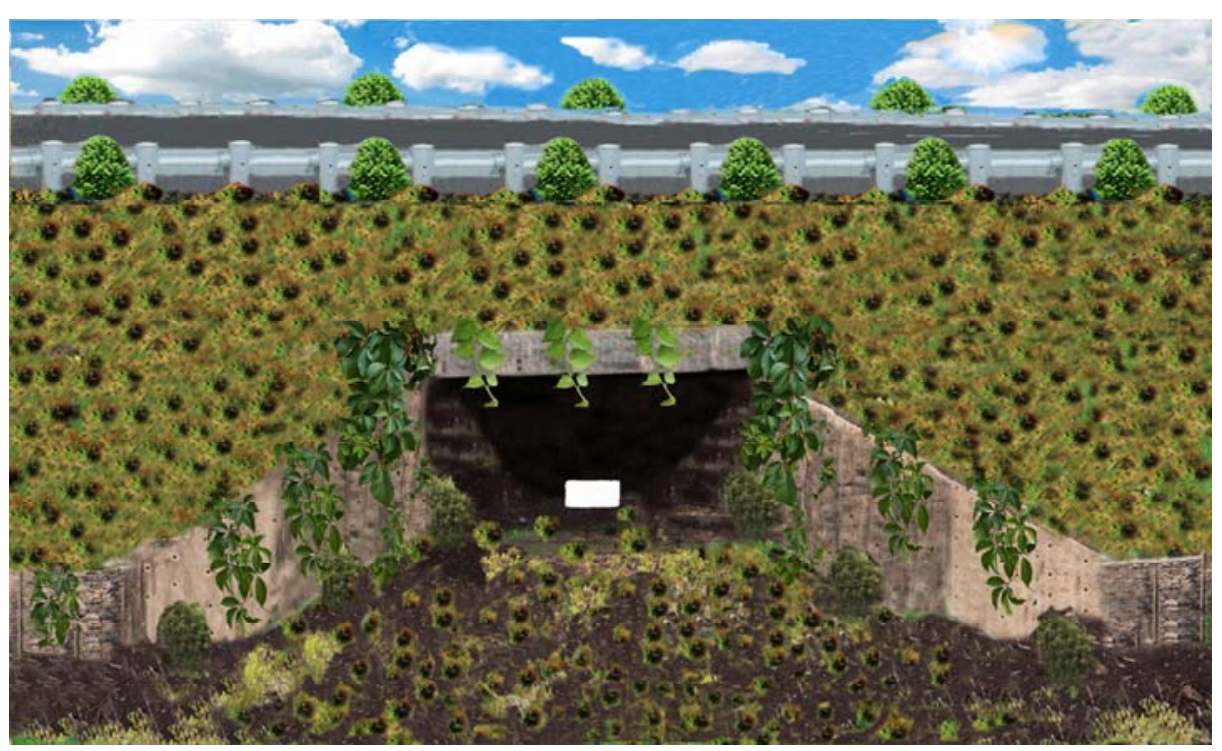

附录2 下钻式野生动物通道效果示意图

Appendix 2 Schematic diagram of wildlife underpass 